The Influence of the Place Value System on Symbolic Number Perception

\author{
Yvonne Oberholzer ${ }^{1}$ \\ Marcus Lindskog ${ }^{2}$ \\ Benjamin Scheibehenne ${ }^{1}$
}

${ }^{1}$ Karlsruhe Institute of Technology, Kaiserstraße 89-93, 76133 Karlsruhe, Germany

${ }^{2}$ Tobii AB, Karlsrovägen 2D, Box 743, S-182 17, Danderyd, Stockholm, Sweden

Corresponding author: Yvonne Oberholzer, yvonne.oberholzer@kit.edu

Data, Materials, and Preregistration on OSF: https://osf.io/pjb7n/

29.06 .2022

Word Count: 5,001 


\begin{abstract}
Past research on symbolic number perception has shown that children's estimates in standard ruler tasks (i.e., placing numbers on a ruler in reference to a start point and an endpoint) follow a logarithmic function. This finding can be explained by assuming that numbers are mapped onto a compressed mental analogue representation. However, two sets of findings are not consistent with this explanation: The different shape of compression for symbolic and non-symbolic numbers and the different developmental change in the two formats. To address these inconsistencies, we endorse an alternative explanation for the logarithmiclooking estimates in children: Misunderstanding of the decimal place value system. To investigate this, we placed adult participants $(N=188)$ in an environment that mimics children's experience with numbers by asking them to do a ruler task with unfamiliar base-26 and base-5 scales. A model comparison (power, linear, logarithmic) revealed that adults showed systematic, logarithmic-looking underestimation on both scales, indicating that the place value system itself can cause the pattern. Additionally, the observed shape of participants' estimates on both scales could be well explained by a place value model that assumes insufficient understanding of exponential growth (i.e., a characteristic of place value systems). Taken together, our results suggest that the logarithmic compression in symbolic number perception does not require the assumption of a compressed shared mental analogue representation but can be explained by the influence of the place value system.
\end{abstract}

Keywords: Place Value, Symbolic Number Perception, Logarithmic Compression, Exponential Growth 


\section{The Influence of the Place Value System on Symbolic Number Perception}

Symbols can be used to communicate numerical information. A common way to express symbolic numbers are Hindu-Arabic numerals, which use a base-10 system including a zero element. However, there are also other number systems that use different symbols or bases. For example, the Roman system employs letters as numerals (e.g., VII =7), and the binary system uses a base of 2 (e.g., $111=7$ ). Beyond these symbolic numbers, quantity can also be communicated non-symbolically for example through dot clouds, which are often used to study cognition in humans and animals. For an overview of different ways to represent number see Table 1.

\section{Table 1}

Comparison of Different Number Notations

\begin{tabular}{cccc}
\hline & Symbolic & & Non-Symbolic \\
\hline Hindu-Arabic & Roman & Binary & Dots \\
\hline 2 & II & 10 & $\bullet \bullet$ \\
4 & IV & 100 & $\bullet \bullet \bullet$ \\
10 & X & 1010 & $\bullet \bullet \bullet \bullet \bullet$
\end{tabular}

Note. Hindu-Arabic, Roman and Binary numbers are a form of symbolic numbers, while dot clouds are a form of non-symbolic numbers. The elements in each row denote the same quantity.

Both perception of symbolic numbers (e.g., Dehaene, 1992; Nuerk et al., 2001; Siegler \& Opfer, 2003; Booth \& Siegler, 2008; Moeller et al., 2009; Berteletti et al., 2010; Barth \& Paladino, 2011) and non-symbolic numbers (e.g., Indow \& Ida, 1977; Krueger, 1972, 1982, 1984; Dehaene, 1992; Ansari, 2008; Izard \& Dehaene, 2008; Dehaene et al., 
2008; Paul et al., 2022) has been studied extensively. While the two evidently share many aspects, such as processing (see Dehaene, 1992), there are also important differences. In the following, we will focus on symbolic number perception.

Past research on symbolic number perception showed that children give more space to smaller numbers on a ruler (Siegler \& Opfer, 2003). More specifically, the children in the study were asked to place different numbers on a ruler going from 0 to 100 or from 0 to 1,000. The second graders (around eight years old) in the study set the midpoint of a scale from 0 to 1'000 to about 130. Similarly, the fourth graders (around 10 years old) set the midpoints to about 190 . Only the sixth graders were able to place it correctly to about 500 . Furthermore, the best fitting function to describe second and fourth graders' estimates was a logarithmic function, not a linear one. A common way to account for these findings is to assume that children's magnitude representations are logarithmically compressed. This assumption aligns with the classical theory of number perception, which posits the existence of a shared number module on which all notations of number can be mapped (e.g., Dehaene, 1992). More specifically, compression is assumed to happen on the level of this shared mental analogue representation, making all type of number perception compressed.

The idea of a compressed shared mental analogue representation has been corroborated by findings in symbolic and non-symbolic number perception, which have shown that estimates of numerical magnitude tend to be underestimated, or compressed (e.g., Indow \& Ida, 1977; Krueger, 1972, 1982, 1984; Dehaene, 1992; Siegler \& Opfer, 2003; Siegler \& Booth, 2004; Berteletti et al., 2010).

However, two sets of findings are not consistent with the hypothesis of a compressed internal representation for all number formats. First, estimates of symbolic and non-symbolic number magnitude give rise to qualitatively different patterns of data with respect to the shape and the degree of compression. For symbolic numbers, the shape is usually logarithmic 
(e.g., Siegler \& Opfer, 2003; Siegler \& Booth, 2004; Berteletti et al., 2010), while for nonsymbolic numbers it is often a power function (e.g., Indow \& Ida, 1977; Krueger, 1972, 1982, 1984). The two are distinct: In a logarithmic function, the argument ( $x$ ) varies, and the base (b) is fitted to the data: $y=\log _{b}(x)$, while in a power function, the base $(x)$ varies, and the exponent $(b)$ is fitted to the data: $y=x^{b}$. Importantly, a power function with a fitted exponent predicts underestimation of all numbers while a logarithmic function with a fitted base can predict overestimation of small numbers. Additionally, even if one assumes that symbolic number processing entails an additional transduction step before mapping onto an internal mental analogue representation, as suggested by Dehaene (1992), it is assumed that the compression takes place on the level of the mental analogue representation, which is the same for both formats (Dehaene, 1992). Therefore, the theory would not predict different shapes and degrees of compression.

Second, while estimates of non-symbolic number magnitudes are still substantially compressed in adulthood (e.g., Indow \& Ida, 1977; Krueger, 1972, 1982, 1984), estimates of symbolic number magnitudes become linear with age (e.g., Siegler \& Opfer, 2003; Siegler \& Booth, 2004). More specifically, children's estimates become linear around second grade (around 8 years old) in the range of 1-100 (Siegler \& Booth, 2004) and around sixth grade (around 12 years old) in the range of 1-1,000 (Siegler \& Opfer, 2003). In contrast, although non-symbolic magnitude estimates become less noisy with age, they are not less compressed in adults (Huntley-Fenner, 2001; Lemaire \& Lecacheur 2007; Tokita \& Ishiguchi, 2013). If number formats share the same compressed internal representation, it is unclear why there would be a developmental change in one format but not the other.

To address these problems, we endorse an alternative explanation why compression in children looks logarithmic based on the decimal place value system (see also Moeller et al., 2009; Siegler \& Opfer, 2003). The decimal system is a characteristic of symbolic numbers, 
and is based on the principle of place value, which makes it possible to express large magnitudes with only a few symbols. More specifically, symbolic numbers expressed in the decimal system have three key properties: (1) the number of elements (figures) used to represent a number: For example, 0-9 are represented with one element and 10-99 with two; (2) the type of element used to represent numbers: The decimal system uses 10 numerals $(0-$ 9) in a defined order; and (3) the place value of an element: For example, a 1 placed to the left of another numeral, as in 10, represents a larger number than a 1 that stands alone. Importantly, in the decimal system, the value of a numeral increases by a factor of 10 for every element that is placed to the right of it (e.g., 1, 10, 100, 1,000).

While the first two properties (number and type of element) are relatively easy to master (e.g., Kaufman et al., 1949; Strauss \& Curtis, 1981; Mandler \& Shebo, 1982; Mix et al., 2014; see also Smyth \& Ansari, 2017), the concept of place value is more challenging. To master it, one has to appreciate that a symbol has to be evaluated in its context (i.e., that the value of a numeral increases by a factor of 10 if it is located to the left of another number). Furthermore, one has to develop an intuition for how the number space expands with each additional numeral (i.e., that it increases exponentially with every additional symbol; see Figure 1). The latter seems difficult to grasp, even for adults (Chesney \& Matthews, 2013). For example, Chesney and Matthews (2013) found that adults' estimates of numerical magnitude become nonlinear again if they are presented with a scale containing anchors with which they are unfamiliar.

\section{Figure 1}

Number Space per Number of Figures in a Segment (Decimal System) 


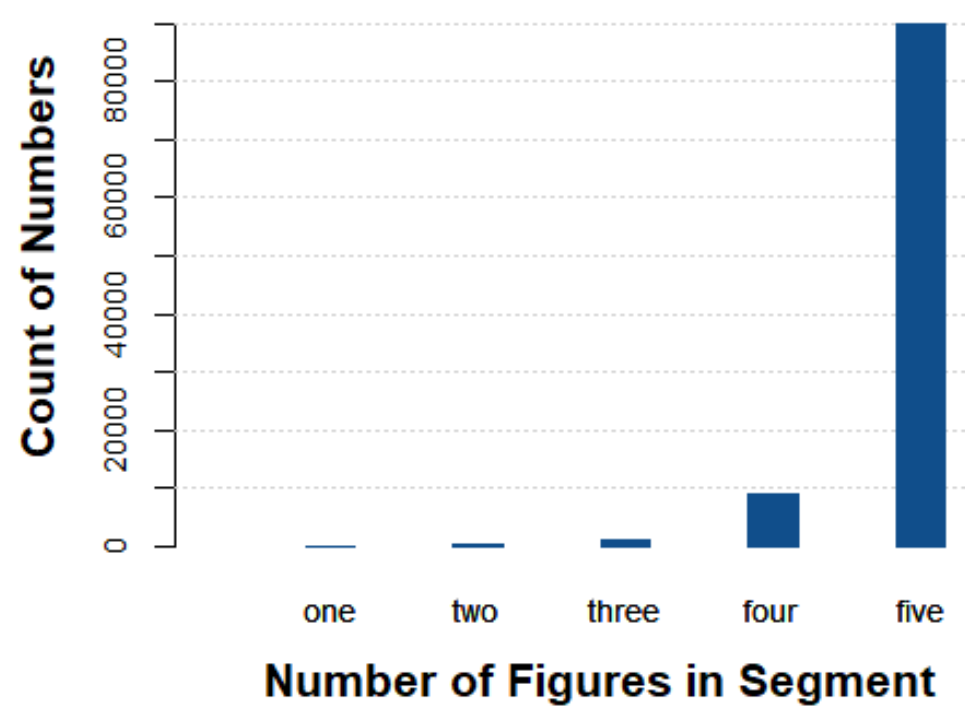

Note. The growth of the decimal number space (i.e., the count of numbers) is depicted based on the number of figures employed in each segment (e.g., the two-figure segment 10-99 contains 9 times as many numbers as the one figure segment $0-10$ ). The image illustrates that the number space increases exponentially with each additional figure. Note that this increase is expressible as $10^{n}-10^{n-1}$, whereby $10^{n}$ is the cumulative count of numbers, and $10^{n-1}$ is the count of the numbers in all the previous segments. Subtracting the count of the previous segment is important to arrive at the number space of the segment in question and not the total count of numbers that make up the scale.

Further support for the idea that the nature of the place value system is difficult to understand comes from research showing that people are notoriously bad at estimating exponential growth (e.g., Wagenaar \& Sagaria, 1975; Wagenaar \& Timmers, 1979; Keren, 1983). In general, people seem to grossly underestimate exponential growth, which is related to poorer investment decisions affecting household finances (Stango \& Zinman, 2009) and suboptimal retirement savings behavior (McKenzie \& Liersch, 2011). Taken together, this indicates that the concept of place value is not easy to master. 
What does this imply? If the decimal system is hard to master and exponential growth is widely underestimated by adults, then misunderstanding of the decimal system can lead to compressed numerical estimates. More specifically, if people falsely assume linear growth for each additional numeral, this leads to logarithmic-looking estimates. Put differently, the place value system of decimal numbers allows for the representation of large numbers in little space, making it a compressed scale itself. Estimates that do not consider this property (i.e., the compressed scale) are bound to look systematically distorted regardless of how an underlying mental representation might look like.

To investigate the influence of place value knowledge on judgments of magnitude, we conducted an experiment, outlined in detail below, in which adults were confronted with unfamiliar place value systems (base-5 \& base-26) in a ruler task. We tested adults, instead of children, because we wanted to make sure that any pattern in our data was specifically due to unfamiliarity with the place value system and not due to a more general lack of knowledge about numbers. The aim was to provide adults with an environment that is highly similar to what children experience when they are not yet familiar with decimal numbers. By presenting adults with number scales that they are not familiar with, we can determine the influence of the place value system on their magnitude judgments. To achieve this, adult participants were presented with a number range $(0-20,000)$ that they were familiar with but with a place value system that they were unfamiliar with as it used a base of either 5 or 26 . Importantly, these systems followed the same principles as the decimal system but used different types and numbers (i.e., figures) of elements to represent the increments. Analogous to numbers, the increments in the unfamiliar systems were interval scaled. Secondly, instead of numerals, the elements consisted of letters from the English alphabet, the form and order of which were familiar to all of our participants, even though they are not usually used to represent number (e.g., a scale going from A to ZZZ). The use of letters helped impede the use of basic 
arithmetic, as basic operations (e.g., multiplication) cannot be easily transferred to letters. This corresponds to children's experience who have not yet mastered basic arithmetic. Additionally, both the alphabet and numbers are usually learnt by children in sequence by counting (e.g., 1, 2, 3, etc., and A, B, C, etc.), making letters a suitable substitute for numerals. In sum, the adults were confronted with symbolic numbers that were based on unfamiliar place value systems and on which they could not do arithmetic easily.

We expected that adults, who theoretically have a linear representation of symbolic magnitude (e.g., Siegler \& Opfer, 2003), would show logarithmically compressed estimates on these symbolic number systems according to the nature of the place value system. This means that an incorrect appreciation of the properties of the place value system could systematically influence number estimates in adults, same as in children (Moeller et al. 2009). Additionally, such an observation would corroborate the explanation for the children's logarithmic estimates (Moeller et al. 2009): difficulty with understanding the place value concept in the decimal system, rather than a compressed internal representation of numbers.

\section{Theoretical Predictions}

On the basis of the theoretical approaches mentioned above, we can derive three distinct predictions for adults' magnitude estimates on unfamiliar place value systems. First, if the compression of symbolic and non-symbolic numbers happens on the level of the shared mental analogue representation (e.g., Dehaene, 1992), we would expect a power-function compression as has been found consistently in adults' non-symbolic number perception (e.g., Indow \& Ida, 1977; Krueger, 1972, 1982, 1984). Second, if adults learn to adopt a more correct linear representation of symbolic numbers (developmental shift, e.g., Siegler \& Opfer, 2003 ) independent of place value knowledge, we would expect them to show a noncompressed linear estimation pattern. Third, if misunderstanding of the place value system leads to systematically biased estimates (Moeller et al., 2009), we would expect 
logarithmic compression in adults confronted with an unfamiliar place value system.

Furthermore, if adults' estimates are based on the misconception that there is linear growth with each additional figure, we can derive a sequential-linear model that can predict adults' magnitude estimates on all unfamiliar place value scales (see also Moeller et al., 2009, for a similar model; and Barth \& Paladino, 2010, for a different model of the ruler task).

To test these different predictions, we compared a power, a linear, a logarithmic, and our place value model on their predictive performance for adults' magnitude estimates in our experiment. The base models for the decimal number range 0-1,000 are depicted in Table 2 .

\section{Table 2}

Base Models and Corresponding Theoretical Predictions

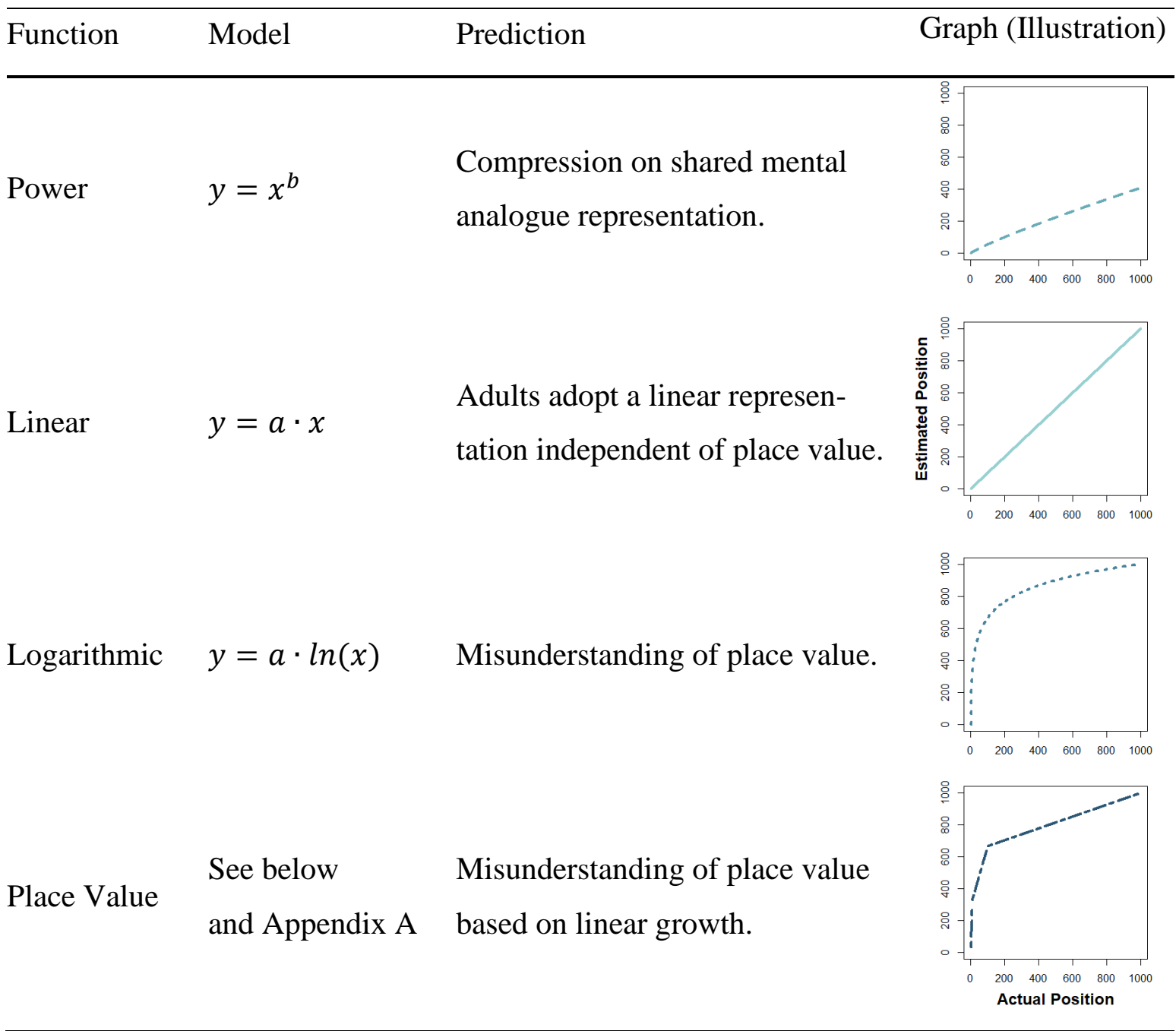


Note. For the logarithmic model, the scaling factor (of the natural logarithm), not the base is modelled because it is easier to implement but offers the same flexibility (i.e., the base $(b)$ of the logarithm $\log _{b}(x)$ can be derived from the scaling factor $(a)$ of the natural logarithm $a$. $\left.\ln (x): b=e^{\frac{1}{a}}\right)$. The graphs are employing values from the literature for illustrative purposes and are based on a ruler task in the number space 1-1,000 with decimal numbers. The power model has an exponent $(b)$ of 0.87 (Indow \& Ida, 1977), the logarithmic model has a scaling factor $(a)$ of $\frac{1}{0.0069}$ (Siegler \& Opfer, 2003) and the linear model has a scaling factor $(a)$ of 1.

We derived the aforementioned place value sequential-linear model based on the assumption that a common misconception of the decimal system is that there is linear growth based on the number of figures instead of exponential growth. More specifically, the place value model states that in a place value system without a zero element (as in the alphabetical system employed in this study), the indices at the point after an element has been added (e.g., AA, AAA, AAAA, etc.) are called switch points (see also Moeller et al. 2009). The position of these switch points indicates the transition between 1-, 2-, and ... n-figure segments and can be described by a cumulative power function of the base $b$ :

$$
s p_{i}=\sum_{1}^{i} b^{i}
$$

where $i$ indicates the index of the specific switch point $s p$. If linear growth is assumed, the estimated switch points esps are described by a function of the endpoint $e p$ and $n$, which denotes the number of elements (i.e., figures) in the endpoint of the scale (e.g., for the base-5 alphabetical system: AAAAA has $n=5$ ).

$$
\operatorname{esp}_{i}=i \cdot \frac{e p}{n}
$$


This function allocates the space equally to the number space between the switch points (see Appendix A or OSF: https://osf.io/sqazk for the full, preregistered outline of the model). We expected our model to capture the observed numerical estimations in our experiment without the need to be fitted to the data (i.e., zero free parameters).

\section{Method}

\section{Material}

The experiment was preregistered on OSF (https://osf.io/sqazk). Both the experiment and the analyses were conducted in line with the preregistration except for (1) the recruitment of five additional participants to adhere to the procedures of the questionnaire host (Prolific), which fills in additional participants if submissions of previous participants have been rejected due to substantial flaws in the data, (2) the use of a power model in which the exponent is fitted (we mistakenly preregistered a power model in which the scaling factor is fitted), and (3) the employment of binomial tests instead of $t$ tests (or Wilcoxon tests) in the analysis as the data violated the assumptions for the intended analyses.

Two different scales were presented to the participants, one base-26 scale ranging to three figures (A-ZZZ) and one base-5 scale ranging to five figures (A-EEEEE). We administered the base- 5 scale as an active control, to guarantee that potentially compressed estimates by the participants were not simply due to the base- 26 scale being more compressed than the standard decimal (base-10) scale. The participants were asked to place nine different indices (e.g., KY) on the scales (see Figure 2).

\section{Figure 2}

\section{Task Example}




\section{Estimation Task 1 / 9}

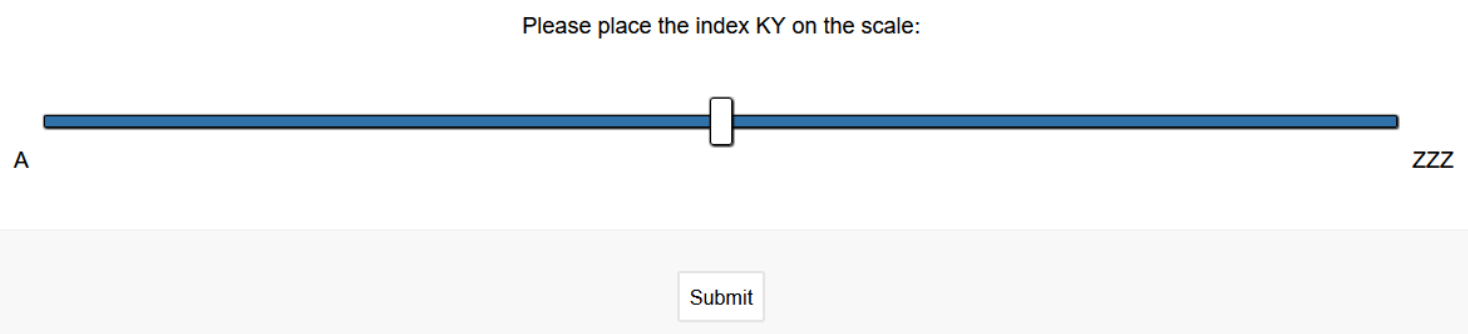

Note. Participants could drag and drop the slider to the estimated position. Their answers were saved after clicking on the "Submit" button. There was no time limit for the task.

We selected nine indices because we wanted to avoid training effects, and a similar number of stimuli were sufficient to distinguish between different models in the past (e.g., Siegler \& Opfer, 2003; Anobile et al., 2012; Slusser et al., 2013). The indices were drawn randomly for each participant from nine bins that spanned the length of the scale. As predictions are more volatile in the lower number space, this space was slightly overrepresented, with the bins on the scale $[0,1]$ being separated at positions $0.01,0.02,0.05$, $0.10,0.2,0.4,0.6$, and 0.8 . In the instructions, it was explained that the scales go through the different letters of the alphabet to the respective endpoint ( $\mathrm{Z}$ or $\mathrm{E})$, after which an additional letter will be added to the right to allow the scale to continue (see Appendix B for the full instructions, including the instruction and attention checks). Four instruction checks were employed to check the participants' understanding of the scale. Additionally, one attention check was employed to check if participants had read the instructions. The design was a between-subjects design to counter carry-over effects. The participants were randomly assigned to one of the two conditions.

\section{Participants and Procedure}


We recruited a total of 245 participants over Prolific according to a preregistered power analysis and inclusion criteria. The study lasted $5.6 \mathrm{~min}$ on average and the participants were paid $£ 0.75$ for participation. Additionally, the participants had the opportunity to enter a tombola, in which we raffled $£ 5.00$ to a participant. The instruction checks could be retaken after failing up to five times, with the instructions being displayed again to the participants. The attention check could not be retaken. The experiment was built in the lab.js editor (Henninger et al., 2019) and distributed via JATOS software (Lange et al., 2015).

\section{Analyses}

Data exclusion was conducted according to the preregistered criteria. Data from seven participants was excluded because the participants indicated that their data should not be used. Additionally, data from 11 participants was excluded due to failing the attention check, and data from 28 participants was excluded due to failing the instruction checks more than once, indicating insufficient understanding of the scale. Finally, data from one participant was excluded for placing the index at the ends or the middle of the scale more than three times, indicating non-serious task completion, and data from 10 participants was excluded for containing more than three intransitivities (non-monotonically increasing) estimates, indicating misunderstanding of the scale (e.g., alphabetical ordering instead of numerical). The final sample included 188 participants, 58\% female, mean age $M=36.0(S D=14.6)$.

\section{Model Prediction Comparison}

Statistical inferences were drawn from a model comparison of the aforementioned models (power, linear, log, place value), each reflecting a distinct theoretical prediction. The predictive performance of each model was specified as the mean out-of-sample root mean squared error (RMSE) yielded by a cross validation (CV) (100× fivefold). A fivefold CV splits the data into training (4/5) and test (1/5) data. The models (except the place value 
model) were fitted to the training data and tested on the test data, with each part set aside as test data once. Compared to previous approaches in which models were fitted to group medians, comparing model predictions based on a CV has the benefit of taking advantage of the full data and allowing for the out-of-sample predictive performance of models (avoiding overfitting; e.g., de Rooij \& Weeda, 2020). Importantly, other model quality estimators such as AIC and BIC are not applicable as the non-fitted place value model does not have a likelihood function. To estimate the predictive performance, we fitted hierarchical versions of the models to the participants' estimates in the training data in R (R Core Team, 2020) using the brms package (Bürkner, 2018). Multilevel-models are needed to account for the repeatedmeasures structure of the data (e.g., Singmann \& Kellen, 2019). Consequently, random slopes were implemented for participant identification (ID) to account for the repeated measures. Because of the task structure, the intercepts were fixed at zero (elements could not be placed off the left end of the ruler). The logarithmic and linear models were estimated with default priors, and the power model was estimated with a weakly informative normal prior with mean 1 and standard deviation 2 (as there are no default priors for nonlinear models). The group-level estimates of the models were then used to predict the test data on the individual level. Eventually, the RMSE on the individual level was averaged over the 100 repetitions of the CV. To be precise, an individual's (j) RMSE was derived as follows:

$$
R M S E_{j}=\sqrt{\frac{\sum_{i=1}^{9}\left(y_{i}-\hat{y}_{i}\right)^{2}}{9}}
$$

where $i$ indicates the index of the observation, $y_{i}$ indicates the observation for that index and $\hat{y}_{i}$ the prediction of the model. For the iterations $c$ of the cross validation, the individual root mean squared errors $\left(R M S E_{j, c}\right)$ are calculated and then averaged to $\overline{R M S E}_{j}$ as follows:

$$
\overline{R M S E}_{j}=\frac{\sum_{c=1}^{100} R M S E_{j, c}}{100}
$$


In contrast to the other models, the place value model did not need to be fitted and was simply used to predict participant estimates. Because of the distribution of the data (nonnormality, unequal variances, and asymmetric distribution of paired differences), we employed binomial tests to analyze the prediction performance of the models using the BayesFactor package (Morey \& Rouder, 2018). Note, a binomial test tests the difference between two groups that contain binary outcomes $(0,1)$. In our analysis, the binary outcome indicates which model had the smaller error in predicting an individual participant's estimates.

\section{Results}

To provide an overview of the data, Figure 4 shows all individual estimates along with the place value model's predictions and the hierarchical models' fits based on the whole data set. To measure the type and degree of compression in both tasks while accounting for out-of-sample error, we first compared the three hierarchical models that were fitted to (training) data in the CV. Figure 5 shows a summary of this comparison by displaying the error each model had in predicting an individual participants' estimates. Table 3 (log columns) summarizes the results of the binomial tests (i.e., count data of how often the model in the column fitted better than its comparison model in the row). In line with our hypothesis, the logarithmic model made better predictions than the other two models in both tasks $\left(\mathrm{BF}_{+} \mathrm{O}\right.$ $>1,000, p<.001$, directional hypothesis, one-sided tests, as preregistered).

\section{Figure 4}

Individual Estimates and Whole Model Fits on the Base-26 and the Base-5 Scales 


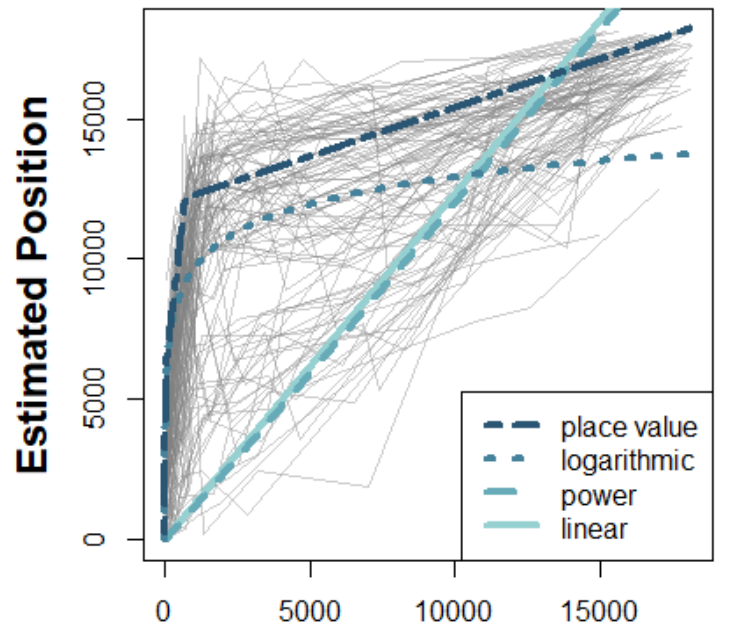

Actual Position

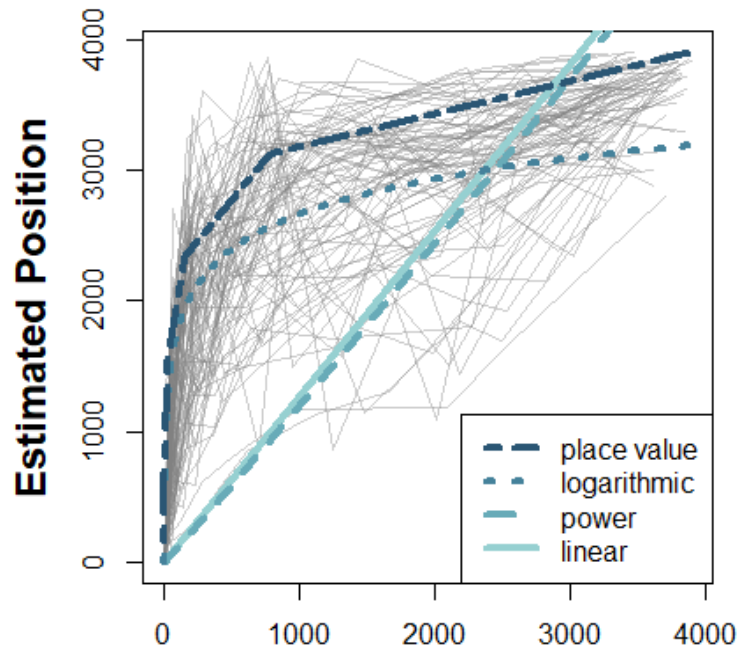

Actual Position

Note. Left panel: Base-26 scale; right panel: base-5 scale. Depicted are individual estimates and model fits (not predictions based on cross validation) based on the whole data set for illustrative purposes. A hierarchical logarithmic, hierarchical power, and hierarchical linear model were fit for the estimates in both scales. The place value model did not need to be fitted to the data. The figure shows that the power and the linear model overlap and that the power model cannot fit the overestimation of small values in the data.

To assess the predictive power of the place value model, we compared it to the three hierarchical models that were fitted to (training) data in the $\mathrm{CV}$. We expected the place value model to make out-of-sample predictions that were as good as or better than those of the logarithmic, the power, and the linear model. Again, the comparison of errors is summarized in Figure 5 and the results of the binomial tests are reported in Table 3 (SM columns). In line with our hypothesis, the place value model predicted the data as well as the logarithmic model in both tasks (base-26: $\mathrm{BF}_{10}=0.47, p=.28$, base-5: $\mathrm{BF}_{10}=1.51, p=.07$, nondirectional hypothesis, two-sided tests, as preregistered), and better than the power and the linear model in both tasks $\left(\mathrm{BF}_{1 \mathrm{O}}>1,000, p<.001\right.$, nondirectional hypothesis, two-sided tests, as preregistered). Finally, as can be seen in Figure 5, the place value model fitted the 
most participants best in the base-26 task $(44.1 \%)$ and the logarithmic model fitted the most participants best in the base- 5 task $(51.2 \%)$.

\section{Figure 5}

$\overline{R M S E}_{j}$ per Model in the Base-26 and the Base-5 Task
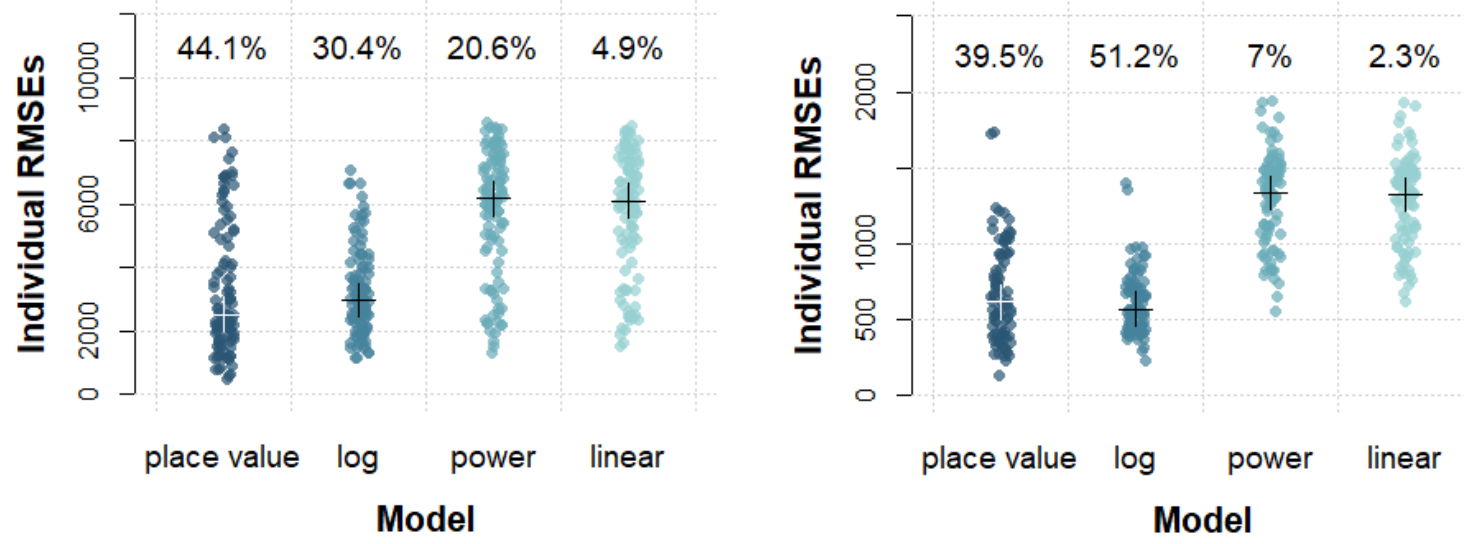

Note. Left panel: Base-26 task; right panel: base-5 task. Depicted are the results of the cross validation $(\mathrm{CV})$. Mean root mean squared error $\left(\overline{R M S E}_{j}\right)$ per participant for each model based on the $100 \times$ fivefold CV for both scales. Medians are indicated with a + . Percentages indicate the proportion of participants best fit by each model (e.g., in the base-26 task, the place value model fitted $44.1 \%$ of the participants best).

\section{Table 3}

Proportion of Participants Best Predicted by the Logarithmic and Place Value Model, Respectively, Compared to the Logarithmic, Power, and Linear Models in the Two Conditions

\begin{tabular}{lllll}
\hline Comparison model & \multicolumn{4}{c}{ Condition } \\
\cline { 2 - 5 } & \multicolumn{2}{c}{ Base-26 $(n=102)$} & \multicolumn{2}{c}{ Base-5 $(n=86)$} \\
\cline { 2 - 5 } & Log & PVM & Log & PVM \\
\hline Log & - & $44 \%$ & - & $40 \%$
\end{tabular}




\begin{tabular}{lcccc}
\hline Comparison model & \multicolumn{3}{c}{ Condition } \\
\cline { 2 - 5 } & \multicolumn{3}{c}{ Base-26 $(n=102)$} & Base-5 $(n=86)$ \\
\cline { 2 - 5 } & Log & PVM & Log & PVM \\
\hline Power & $75 \%^{\mathrm{a}, * * *}$ & $72 \%^{\mathrm{a}, * * *}$ & $92 \%^{\mathrm{a}, * * *}$ & $80 \%^{\mathrm{a}, * * *}$ \\
Linear & $75 \%^{\mathrm{a}, * * *}$ & $72 \%^{\mathrm{a}, * * *}$ & $91 \%^{\mathrm{a}, * * *}$ & $80 \%^{\mathrm{a}, * * *}$ \\
\hline
\end{tabular}

Note Log = Logarithmic model; PVM = place value model. The percentages indicate the proportion of participants predicted better by the model in the column than model in the row. Letters indicate substantial evidence in favor of the alternative hypothesis, BF $>10$. Asterisks indicate significant effects at $\alpha=.05$.

${ }^{\mathrm{a}} \mathrm{BF}>1,000$.

$* * * p<.001$.

\section{Discussion}

Previous research suggested that the compressed nature of non-symbolic and symbolic number estimates alike arises from compression on the level of the shared mental analogue representation (Dehaene, 1992). Furthermore, it has been suggested that a developmental shift occurs during which children learn to adopt a non-compressed, linear representation of numbers (Siegler \& Opfer, 2003). In the current study, we tested an alternative hypothesis predicting that the logarithmic shape of symbolic number estimates arises from a misconception of the exponential nature of the decimal system (Moeller et al., 2009). In the effort to isolate a potential effect of the nature of the place value system, we tested adults rather than children.

In line with our predictions, the adult participants' estimates on both unfamiliar scales were neither shaped like a power function nor linear but instead were better predicted by a logarithmic function. Additionally, the place value model, which assumes linear growth based on the number of figures, was able to predict the data remarkably well; for both scales, 
the predictions of the place value model were as good as or better than the predictions of the logarithmic, the power, and the linear model.

These results suggest that adults do not have a linear understanding of symbolic magnitude independent of the place value system in which it is represented. If adults were able to generalize their knowledge from the decimal system, they should have placed the indices on the scale in a more linear fashion. Furthermore, in contrast to what would be expected if symbolic numbers were mapped onto the same mental analogue representation as non-symbolic numbers, our results also indicate that adults do not have a power-functionbased representation of symbolic magnitude. If they did, they would show power-functionbased compression of their estimates and the power model would have made more accurate predictions for both scales.

Instead, our results show that, ruler-based magnitude judgments of adults depend on the format of the place value system, mirroring what has been found in children (Moeller et al., 2009). Moreover, as can be seen in Figure 4 (axes), the compression of estimates on the two different scales is strikingly different. This is also reflected in the best fitting beta coefficients of the full logistic hierarchical multilevel model (base-26: $\beta=1,403,95 \%$ Credible Interval (CrI) $[1,346,1,460]$; base-5: $\beta=386,95 \% \mathrm{CrI}[374,398])$. This finding is consistent with previous research indicating different rates of compression when different ranges of the decimal system are used (e.g., Siegler \& Opfer, 2003). Thus, if symbolic numbers are indeed mapped onto an internal mental analogue representation, the mapping would have to have different properties depending on the range presented to participants. A more parsimonious explanation is, in our view, that the format of the place value system systematically influences magnitude perception. This explanation is further supported by the finding that our place value model provided an adequate fit to the data. Furthermore, that the results in the two scales (base-26 and base-5) were equivalent in terms of the shape of the 
compression shows that participants did not simply show compressed estimates because the base-26 scale is more compressed than the standard decimal scale (base-10).

Taken together, our results indicate that the difference in compression between symbolic and non-symbolic numbers can be explained by the influence of number format (i.e., dots vs decimal place value notation). Furthermore, these results also provide an explanation for why there is a developmental change in symbolic numbers, but not in nonsymbolic numbers. As proposed by Moeller and colleagues (2009), and speculated by Siegler and Opfer (2003), the shift can be interpreted as a mastery of the understanding of the decimal place value system. We speculate that the shift might coincide with the introduction of multiplication and division in school, during which children come to appreciate the exponential nature of the number system when they learn multiplication and division by the corresponding factors (e.g., 10). More specifically, division might play an important role as it allows for making relative and proportional judgments based on the scale endpoint (e.g., the endpoint divided by 2 equals the magnitude at the midpoint; see Barth \& Paladino, 2011; Ashcraft \& Moore, 2012). Future research could address how the mastery of the decimal system, and multiplication and division arithmetic relate to the shape of magnitude estimates in children. Similarly, potential intervention studies could reveal whether place value knowledge could be improved even in adults.

There are a number of limitations to the current study: First, data attrition was slightly higher for the base-5 scale than for the base-26 scale. A possible reason for this is that participants found it more difficult to understand the base-5 scale. The increased difficulty might be due to (a) the scale ending at $\mathrm{E}$, which is not a natural endpoint, such as Z, for our English-speaking participants or (b) the number of letters in an index, which was larger for the base- 5 scale than the base-26 scale, making mix-ups more likely (e.g., mistaking AAAAA for AAAA). 
Second, even though we excluded participants showing inconsistent estimates according to our preregistered criteria (e.g., not adhering to the order $\mathrm{A}-\mathrm{Z}$ of indices), there were still some participants with rather noisy and partly intransitive estimates, as can be seen in Figure 4. This might have introduced some noise in the model comparison.

Third, as Figure 4 reveals, no single model was able to predict all participants well. This seems to indicate that there are individual differences in how well people cope with unfamiliar place value systems. Future research could potentially investigate mechanisms that drive these differences.

\section{Conclusion}

In summary, our study shows that unfamiliar place value systems can systematically shape numerical estimates in adults, making them look logarithmic in shape. We conclude that insufficient understanding of place value (e.g., in the decimal system) provides a parsimonious explanation for the observed discrepancy between symbolic and non-symbolic number perception and potentially also the developmental shift from logarithmic to linear estimates in children.

\section{CRediT statement}

Yvonne Oberholzer: Conceptualization, Data Curation, Formal Analysis, Investigation, Methodology (lead), Project Administration, Resources, Software, Validation, Visualization, Writing - Original Draft, Writing - Review \& Editing. Benjamin Scheibehenne: Conceptualization, Funding Acquisition, Methodology (supporting), Supervision, Writing - Review \& Editing. Marcus Lindskog: Conceptualization, Methodology (supporting), Supervision, Writing - Review \& Editing.

\section{Acknowledgments}

This research has been funded by the Swiss National Science Foundation (SNSF) Grant Nr. 100014_175849. We thank Anita Todd for editing the manuscript. 


\section{Supplemental Material}

The experiment, the data and the analysis are available on the OSF project page (https://osf.io/pjb7n/). 


\section{References}

Anobile, G., Cicchini, G. M., \& Burr, D. C. (2012). Linear mapping of numbers onto space requires attention. Cognition, 122(3), 454-459. https://doi.org/10.1016/j.cognition.2011.11.006

Ansari, D. (2008). Effects of development and enculturation on number representation in the brain. Nature Reviews Neuroscience, 9(4), 278-291. https://doi.org/10.1038/nrn2334

Ashcraft, M. H., \& Moore, A. M. (2012). Cognitive processes of numerical estimation in children. Journal of Experimental Child Psychology, 111(2), 246-267. https://doi.org/10.1016/j.jecp.2011.08.005

Barth, H. C., \& Paladino, A. M. (2011). The development of numerical estimation: Evidence against a representational shift: Development of numerical estimation. Developmental Science, 14(1), 125-135. https://doi.org/10.1111/j.1467-7687.2010.00962.x

Berteletti, I., Lucangeli, D., Piazza, M., Dehaene, S., \& Zorzi, M. (2010). Numerical estimation in preschoolers. Developmental Psychology, 46(2), 545-551. https://doi.org/10.1037/a0017887

Booth, J. L., \& Siegler, R. S. (2008). Numerical magnitude representations influence arithmetic learning. Child Development, 79(4), 1016-1031. https://doi.org/10.1111/j.1467-8624.2008.01173.x

Bürkner, P.-C. (2018). Advanced Bayesian multilevel modeling with the R package brms. The R Journal, 10(1), 395-411.

Chesney, D. L., \& Matthews, P. G. (2013). Knowledge on the line: Manipulating beliefs about the magnitudes of symbolic numbers affects the linearity of line estimation tasks. Psychonomic Bulletin \& Review, 20(6), 1146-1153. https://doi.org/10.3758/s13423013-0446-8 
Dehaene, S. (1992). Varieties of numerical abilities. Cognition, 44(1), 1-42. https://doi.org/10.1016/0010-0277(92)90049-N

Dehaene, S., Izard, V., Spelke, E., \& Pica, P. (2008). Log or linear? Distinct intuitions of the number scale in Western and Amazonian indigene cultures. Science, 320(5880), 12171220. https://doi.org/10.1126/science. 1156540

de Rooij, M., \& Weeda, W. (2020). Cross-validation: A method every psychologist should know. Advances in Methods and Practices in Psychological Science, 3(2), 248-263. https://doi.org/10.1177/2515245919898466

Henninger, F., Shevchenko, Y., Mertens, U. K., Kieslich, P. J., \& Hilbig, B. E. (2019). lab.js: A free, open, online study builder [Preprint]. PsyArXiv. https://doi.org/10.31234/osf.io/fqr49

Huntley-Fenner, G. (2001). Children's understanding of number is similar to adults' and rats': Numerical estimation by 5-7-year-olds. Cognition, 78(3), B27-B40. https://doi.org/10.1016/S0010-0277(00)00122-0

Indow, T., \& Ida, M. (1977). Scaling of dot numerosity. Perception \& Psychophysics, 22(3), 265-276. https://doi.org/10.3758/BF03199689

Izard, V., \& Dehaene, S. (2008). Calibrating the mental number line. Cognition, 106(3), 1221-1247. https://doi.org/10.1016/j.cognition.2007.06.004

Kaufman, E. L., Lord, M. W., Reese, T. W., \& Volkmann, J. (1949). The discrimination of visual number. The American Journal of Psychology, 62(4), 498-525. https://doi.org/10.2307/1418556

Keren, G. (1983). Cultural differences in the misperception of exponential growth. Perception \& Psychophysics, 34(3), 289-293. https://doi.org/10.3758/BF03202958 
Krueger, L. E. (1972). Perceived numerosity. Perception \& Psychophysics, 11(1), 5-9. https://doi.org/10.3758/BF03212674

Krueger, L. E. (1982). Single judgments of numerosity. Perception \& Psychophysics, 31(2), 175-182. https://doi.org/10.3758/BF03206218

Krueger, L. E. (1984). Perceived numerosity: A comparison of magnitude production, magnitude estimation, and discrimination judgments. Perception \& Psychophysics, 35(6), 536-542. https://doi.org/10.3758/BF03205949

Lange, K., Kühn, S., \& Filevich, E. (2015). "Just another tool for online studies” (JATOS): An easy solution for setup and management of web servers supporting online studies. PLOS ONE, 10(6), e0130834. https://doi.org/10.1371/journal.pone.0130834

Lemaire, P., \& Lecacheur, M. (2007). Aging and numerosity estimation. The Journals of Gerontology. Series B, Psychological Sciences and Social Sciences, 62(6), P305-312. https://doi.org/10.1093/geronb/62.6.p305

Mandler, G., \& Shebo, B. J. (1982). Subitizing: An analysis of its component processes. Journal of Experimental Psychology: General, 111(1), 1-22.

McKenzie, C. R. M., \& Liersch, M. J. (2011). Misunderstanding savings growth: Implications for retirement savings behavior. Journal of Marketing Research, 48(SPL), S1-S13. https://doi.org/10.1509/jmkr.48.SPL.S1

Mix, K. S., Prather, R. W., Smith, L. B., \& Stockton, J. D. (2014). Young Children's Interpretation of Multidigit Number Names: From Emerging Competence to Mastery. Child Development, 85(3), 1306-1319. https://doi.org/10.1111/cdev.12197 
Moeller, K., Pixner, S., Kaufmann, L., \& Nuerk, H.-C. (2009). Children's early mental number line: Logarithmic or decomposed linear? Journal of Experimental Child Psychology, 103(4), 503-515. https://doi.org/10.1016/j.jecp.2009.02.006

Morey, R. D., \& Rouder, J. N., (2018) BayesFactor: Computation of Bayes Factors for Common Designs. R package version 0.9.12-4.2. https://CRAN.Rproject.org/package=BayesFactor

Nuerk, H.-C., Weger, U., \& Willmes, K. (2001). Decade breaks in the mental number line? Putting the tens and units back in different bins. Cognition, 82(1), B25-B33. https://doi.org/10.1016/S0010-0277(01)00142-1

Paul, J. M., van Ackooij, M., ten Cate, T. C., \& Harvey, B. M. (2022). Numerosity tuning in human association cortices and local image contrast representations in early visual cortex. Nature Communications, 13(1), 1340. https://doi.org/10.1038/s41467-02229030-z

R Core Team. (2020). R: A language and environment for statistical computing [Computer software manual]. R Foundation for Statistical Computing. https://www.R-project.org/

Siegler, R. S., \& Booth, J. L. (2004). Development of numerical estimation in young children. Child Development, 75(2), 428-444. https://doi.org/10.1111/j.14678624.2004.00684.x

Siegler, R. S., \& Opfer, J. E. (2003). The development of numerical estimation: Evidence for multiple representations of numerical quantity. Psychological Science, 14(3), 237-250. https://doi.org/10.1111/1467-9280.02438

Singmann, H., \& Kellen, D. (2019). An Introduction to Mixed Models for Experimental Psychology. In New Methods in Cognitive Psychology. Routledge. 
Slusser, E. B., Santiago, R. T., \& Barth, H. C. (2013). Developmental change in numerical estimation. Journal of Experimental Psychology: General, 142(1), 193. https://doi.org/10.1037/a0028560

Smyth, R., \& Ansari, D. (2017). Do infants have a sense of numerosity? A p-curve analysis of infant numerosity discrimination studies. Developmental Science, 23(2), e12897. https://doi.org/10.31219/osf.io/5rcxm

Stango, V., \& Zinman, J. (2009). Exponential growth bias and household finance. The Journal of Finance, 64(6), 2807-2849. https://doi.org/10.1111/j.15406261.2009.01518.x

Strauss, M. S., \& Curtis, L. E. (1981). Infant perception of numerosity. Child Development, 52(4), 1146-1152. https://doi.org/10.2307/1129500

Tokita, M., \& Ishiguchi, A. (2013). Effects of perceptual variables on numerosity comparison in 5-6-year-olds and adults. Frontiers in Psychology, 4. https://doi.org/10.3389/fpsyg.2013.00431

Wagenaar, W. A., \& Sagaria, S. D. (1975). Misperception of exponential growth. Perception \& Psychophysics, 18(6), 416-422. https://doi.org/10.3758/BF03204114

Wagenaar, W. A., \& Timmers, H. (1979). The pond-and-duckweed problem; Three experiments on the misperception of exponential growth. Acta Psychologica, 43(3), 239-251. https://doi.org/10.1016/0001-6918(79)90028-3 


\section{Appendix A}

\section{Outline of the Place Value Model (preregistered as: Simple Model) in Pseudo Code}

The place value model assumes linear instead of exponential growth of the number space for each additional element (numeral). We call the numbers at the point after an element has been added switch points (e.g., for the base-5 alphabetical system: AA, AAA, AAAA, etc.). In a place value system without a zero element (as in the alphabetical system), the switch points $s p$ s for a place value system with base $b$ are described by a cumulative power function of the base:

$$
s p_{i}=\sum_{1}^{i} b^{i}
$$

where $i$ indicates the index of the specific switch point. If linear growth is assumed, the estimated switch points esps are described by a function of the endpoint $e p$ and $n$, which denotes the number of elements at the endpoint of the scale (e.g., for the base- 5 alphabetical system: AAAAA has $n=5$ ).

$$
e s p_{i}=i \cdot \frac{e p}{n}
$$

This function allocates the space equally to the number space between the switch points. For the number space between the switch points, linear functions predicting the estimated number $y$ from the actual number $x$ can be derived in the following way. For the first segment,

$$
y_{1}=0+\frac{e s p_{1}}{s p_{1}} \cdot x
$$

The function starts at intercept 0 and has a slope of $\frac{e s p_{1}}{s p_{1}}$. For every consecutive number space, the linear equation can be derived as follows: 


$$
y_{i}=e s p_{i-1}+\frac{e s p_{i}-e s p_{i-1}}{s p_{i}-s p_{i-1}} \cdot\left(x-s p_{i-1}\right)
$$

The function starts at intercept $e s p_{i-1}$. The slope is defined as the differences in actual and estimated switch points from the present and the previous number space. Finally, $x$ has to be corrected for the numbers already present in the previous number space.

\section{Special Case for a Place Value System With a "Zero" Element}

In a place value system with a zero element ranging from 0 to a multiple of the base, the switch points are instead located at

$$
s p_{i}=b^{i}
$$

In addition, the number of elements $n$ has to be reduced by 1 , as there are fewer switch points. The equation for the estimated switch points and the sequential linear equations are equally applicable. 


\section{Appendix B \\ Experiment Instructions, Instruction Checks, and Attention Check}

The full experiment (.json) including instructions on how to run it are uploaded on OSF (https://osf.io/pjb7n/). Relevant excerpts are reported in this appendix.

\section{Figure B1}

Instructions and Instruction Checks as Presented During the Experiment Instructions:

Imagine a scale that starts at $A$, going through the letters of the alphabet to $Z$. So: $A, B, C, \ldots Y, Z$.

After $Z$, the scale starts again at AA continuing with $A B, A C, A D, A E, B A, B B, B C, \ldots$ and so on until $Z Z$.

Each time the full set has been counted (an $Z$ is changed to an A), the letter to the left is counted up by one. As written above, $A Z$ is followed by BA.

Finally, when there are no letters left to count anymore (all letters are „Z“), another letter is added to the right side. As an example, $\mathrm{ZZ}$ is followed by AAA.

Thereby, the scale eventually goes from A to ZZZ:

A

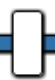

$\mathrm{ZZZ}$

Here are some examples to help you understand the scale:

- After ABC comes ABD.

- After ZZ comes AAA.

- After BAZ comes BBA.

Indices with less letters (e.g. ZZ: 2 letters) will be placed before indices with more letters on this scale (e.g. AAA: 3 letters). In other words, the scale will first go through all the 1-letter indices and then through all the 2-letter indices until it ends at the last 3-letter index. 
To check your understanding of the scale, please answer the following questions correctly.

1. Which index comes after EF?

O EG

$\mathrm{OED}$

$\mathrm{OEC}$

2. Which index comes after MZ?
$O M$
ONA
$O M X$

3. Which index would be placed leftmost on the scale?

$O A B C$

$\mathrm{OHI}$

OW

4. Which index would be placed rightmost on the scale?

OS

O LAX

O GE

In the main task, you will be asked to place different indices on the scale (as shown above).

The default position of the index is in always the middle of the scale. You can adjust the index with the mouse (drag and drop it or click on the scale directly).

Note. The instructions and the instruction checks were presented on the same page. The instruction checks could be retaken up to five times. However, only participants with less than two repetitions (allowing for one careless mistake) were included in the final data. If an instruction check was answered incorrectly, an error message appeared in red on the page, stating the error and asking participants to read the instructions again. Furthermore, the incorrectly answered checks were marked in red too.

\section{Figure B2}

Attention Check as Presented During the Experiment 
Please answer the question below.

According to the instructions that you have just read, what should you do in the following task?

$O$ indicate how much I agree with the statements

$\mathrm{O}$ indicate if the indices contain a "B"

$\bigcirc$ place different indices on a scale

select the correct index from 5 options

Note. The attention check was presented on a separate page after the instructions and the instruction checks. The attention check could not be retaken. In case of failure, a message was displayed to the participants stating the error. The participants could proceed to the task if they wished to do so but were told that their submission might likely be rejected because of their insufficient attention to the task. 